\title{
Effects of IFN- $\gamma$ on the proliferation of 32D cells expressing Akt after IRF-1 gene silencing
}

\author{
Ying Lin ${ }^{1,2}$, Rongdong Zhang ${ }^{2}$, Shenghua Jiang ${ }^{1}$, Wei Lu ${ }^{1}$, Zenghua Lin ${ }^{1 *}$, Hong Liu ${ }^{1 *}$ \\ ${ }^{1}$ Department of Hematology, Affiliated Hospital of Nantong University, Nantong University, Nantong, China; ${ }^{2}$ Department of Hematology, Ningde \\ Hospital affiliated to Medical University of Fujian, Ningde, China \\ Contributions: (I) Conception and design: Y Lin, Z Lin; (II) Administrative support: H Liu; (III) Provision of study materials or patients: R Zhang, S \\ Jiang; (IV) Collection and assembly of data: W Lu, Z Lin; (V) Data analysis and interpretation: Y Lin, Z Lin; (VI) Manuscript writing: All authors; (VII) \\ Final approval of manuscript: All authors. \\ *These authors contributed equally for the senior authorship. \\ Correspondence to: Zenghua Lin; Hong Liu. Department of Hematology, Affiliated Hospital of Nantong University, Nantong University, Nantong \\ 226001, China. Email: linzenghua@126.com; hongliu63@126.com.
}

Background: Interferon regulatory factor-1 (IRF-1) plays a critical role in the injury to stem and progenitor regions associated with aberrant interferon-gamma (IFN- $\gamma$ ) in aplastic anemia (AA). The present study aimed to investigate the effects of IFN- $\gamma$ on murine myeloid precursor cells (32D cells) with wild-type and inactive-type protein kinase B (Akt) after $I R F-1$ gene silencing.

Methods: With treatment of four concentrations of IFN- $\gamma$, the $32 \mathrm{D}$ cell viability and inhibition rate were assayed by middle-time-spray (MTS). The apoptosis rate was determined by flow cytometry, and the expression of the phosphorylated signal transducer and activator of transcription 3 (p-Stat3) and the phosphorylated signal transducer and activator of transcription 5 (p-Stat5) was analyzed by Western blot.

Results: The results from real time PCR (RT-PCR) assays suggested that the relative expression level of IRF-1-mRNA in the knockdown group (KD) was lower than that of in the negative control (NC) and blank control (Ctrl). In addition, the silencing efficiency was $>70 \%$, which was further validated by Western blotting. At $48 \mathrm{~h}$, the rate of proliferation of 32D cells of wild-type Akt was significantly higher than that of inactive-type Akt $(0.918 \pm 0.005$ vs. $0.503 \pm 0.003, \mathrm{P}=0.008)$, while the apoptosis rate in wild-type was significantly lower than that of inactive Akt $(1.46 \% \pm 0.41 \%$ vs. $2.98 \% \pm 0.32 \%, \mathrm{P}=0.006)$. After reducing the expression of $I R F-1$ gene, the promotion of hematopoiesis was recovered, resulting from the high concentration of IFN- $\gamma$ achieved by reducing the expression of $\mathrm{p}$-Stat 5 via the Akt signaling pathway.

Conclusions: Taken together, these results suggested that IRF-1 plays a critical role in the pro-apoptotic effect of IFN- $\gamma$ on the proliferation of hematopoietic progenitor cells. These findings could contribute to understanding the mechanisms underlying the conversion from IFN- $\gamma$-mediated inhibition to promotion of hematopoiesis.

Keywords: Akt; interferon regulatory factor-1 (IRF-1); interferon-gamma (IFN- $\gamma$ ); proliferation; aplastic anemia (AA)

Submitted Apr 18, 2020. Accepted for publication Sep 12, 2020.

doi: $10.21037 /$ tcr-20-1866

View this article at: http://dx.doi.org/10.21037/tcr-20-1866

\section{Introduction}

Aplastic anemia (AA) is characterized by marrow failure due to several reasons. Although the exact pathogenesis is unclear, it is speculated to be caused by immunological relevance (1). The abnormal activation of $\mathrm{T}$ lymphocytes and the excess secretion of interferon- $\gamma(\mathrm{IFN}-\gamma)$ are major factors, resulting in subsequent apoptosis of hematopoietic cells $(1,2)$. The expression of IFN- $\gamma$ is regulated by IFN- $\gamma$ 
gene polymorphism and is influenced by interferon regulatory factor-1 (IRF-1) gene; also, the residual expression of IRF-1 might inhibit the cell proliferation (3-6). Under the condition of downregulated expression of IRF1 , the pro-apoptotic role of IFN- $\gamma$ can be reversed. Our previous study showed that activated Akt can significantly promote the proliferation and inhibit apoptosis of mouse myeloid progenitor cell line (32D cells) and IFN- $\gamma$ can regulate cell proliferation and apoptosis by regulating Akt (7). To date, the article associated with the reversal of IFN- $\gamma$ on the hematopoietic stem/progenitor cells has not reported. Jak-Stat and PI3K/Akt signaling pathways play critical roles in cell proliferation and apoptosis $(8,9)$; however, the pathway underlying the reversal of IFN- $\gamma$ is not yet clarified. Herein, with the effects of IFN- $\gamma$ on 32D cells expressing wild-type Akt after IRF-1 gene silencing, we explored the mechanisms of IFN- $\gamma$ mediated conversion from inhibition to promotion of hematopoiesis and our findings might contribute to a better understanding of the bidirectional regulation of interferon on hematopoiesis. We present the following article in accordance with the MDAR checklist (available at http:// dx.doi.org/10.21037/tcr-20-1866).

\section{Methods}

\section{Cell culture}

Murine myeloid precursor 32D cells were stably transfected with the wild-type Akt and inactive-type Akt (Inactive Akt mutant plasmids were transfected into 32D cells using Lipofectamine 2000 according to the manufacturer's manual), respectively. The cells were provided by F. Dong (Toledo University, OH, USA) as a generous gift. The cells were maintained in Roswell Park Memorial Institute (RPMI) 1640 medium supplemented with $10 \%$ fetal bovine serum (FBS), 10\% WEHI-3B cell-conditioned reagent including IL-3 (essential nutrition cytokine), $100 \mu \mathrm{g} / \mathrm{mL}$ penicillin, and $100 \mu \mathrm{g} / \mathrm{mL}$ streptomycin.

\section{Reagents}

Antibodies against IRF-1, Stat3, phospho-Stat3, Stat5, phospho-Stat5 were purchased from Cell Signaling Technology. Recombinant murine IFN- $\gamma$ was purchased from PeproTech. Interference vector of the $I R F-1$ gene was constructed and packaged by GeneChem (Shanghai, China). The apoptosis detection kit and Lipofectamine 2000 reagent were obtained from Invitrogen. Super ECL Plus Detection Reagent was procured from Pierce Biotechnology.

\section{siRNA vector construction and transfection}

Short interfering RNA (siRNA) eukaryotic expression vector to reduce the expression of $I R F-1$ was constructed and transfected into 32D cells expressing wild-type Akt and inactive-type Akt. According to the requirements of different groups, the cells were grown to the logarithmic growth phase in 24-well plates and transfected with siRNAs according to the instructions of Lipofectamine 2000. Then, the silencing effect of the $I R F-1$ gene was detected by realtime quantitative polymerase chain reaction (RT-qPCR) and Western blotting.

\section{Detection of silencing effect through RT-PCR assays}

Total RNA was extracted by TRIzol, and cDNA was synthesized through reverse transcription according to the instructions of the reverse transcriptase kit in a two-step RT-PCR. The forward primer sequence of $I R F-1$ was GGGACATTGGGATAGGCA and that of reverse was CTCAGGAGGGCAAGAACG. In addition, the forward primer sequence of $G A P D H$ was TGGTGAAGGTCGGTGTGAAC and that of reverse was GCTCCTGGAAGATGGTGATGG. The reaction conditions were as follows: pre-denaturation at $95{ }^{\circ} \mathrm{C}$ for $15 \mathrm{~s}$, followed by 45 cycles of denaturation at $95{ }^{\circ} \mathrm{C}$ for $5 \mathrm{~s}$, annealing and extension at $60{ }^{\circ} \mathrm{C}$ for $30 \mathrm{~s}$. The $\mathrm{Ct}$ values were analyzed to obtain the relative expression of the target gene.

\section{Detection of the cell viability and inbibition rate}

The logarithmic growth phase cells were seeded in 96-well plates and incubated at $37^{\circ} \mathrm{C}$ in $5 \% \mathrm{CO}_{2}$ incubator, followed by addition of the drug on the next day according to the experimental groups. After 24, 48, and $72 \mathrm{~h}$ incubation, 20 $\mu \mathrm{L}$ of middle-time-spray (MTS) reagent was added. The reaction was terminated at $3 \mathrm{~h}$, and OD value was measured at $490 \mathrm{~nm}$ by a microplate reader.

\section{Detection of cell apoptosis}

Cells $\left(1 \times 10^{5}\right.$ cells $\left./ \mathrm{mL}\right)$ were incubated with various concentrations of IFN- $\gamma$ for $24 \mathrm{~h}$ and $48 \mathrm{~h}$, respectively. 
Then, the cells were collected after serum starvation of $4 \mathrm{~h}$ (yet with IL-3), and the apoptotic rate was determined by flow cytometry.

\section{Western blot analysis}

The cells were cultured in the presence of various concentrations of IFN- $\gamma$ after transfection with siRNA vector and negative control (NC) and serum starvation for $4 \mathrm{~h}$. The cell extracts were prepared and separated by SDSPAGE, followed by transfer to a PVDF membrane. The membranes were blocked at room temperature for $2 \mathrm{~h}$ and probed with appropriate antibodies. The immunoreactive proteins were detected by ECL following the manufacturer's protocol.

\section{Statistical analysis}

Statistical significance in our results were calculated using GraphPad software. In this paper, CCK8 and real-time PCR data were analyzed using the two-tailed Student's $t$-test. In general, n.s. indicates not significant, and *, **, and ${ }^{* * *}$ indicate $\mathrm{P}$ values less than $0.05,0.01$, and 0.001 , respectively. All statistical significance was calculated using "knockdown group (KD)" vs. "blank control (Ctrl)", or "negative control (NC)" vs. "blank control (Ctrl)".

\section{Results}

\section{IRF-1 gene silencing through siRNA}

To verify the silencing effect of the $I R F-1$ gene, fluorescence microscopy was used to observe the effect of transfection (Figure 1A). The results from RT-PCR assays suggested that the relative expression level of $I R F-1$-mRNA in the knockdown group (KD) was less than that of in the $\mathrm{NC}$ and blank control (Ctrl) (Figure 1B). In addition, the silencing efficiency was $>70 \%$, which was further validated by Western blotting. The expression level of IRF-1 protein in the $I R F-1$-siRNA group was significantly lower than that of in the NC and Ctrl groups (Figure 1C).

\section{Detection of the cell viability and apoptotic rate after IRF-1 gene silencing}

Cell viability and inhibition rate were assayed by MTS after incubation with IFN- $\gamma$ for up to 6 days, and the apoptotic rate was determined by flow cytometry after incubation for $24 \mathrm{~h}$ and $48 \mathrm{~h}$ and after serum starvation for $4 \mathrm{~h}$. After treatment with IFN- $\gamma$ for $24 \mathrm{~h}$, the low concentration of IFN- $\gamma$ in the $I R F-1$ downregulated group promoted proliferation and inhibited apoptosis, whereas the high concentration suppressed the proliferation and induced apoptosis. Moreover, at $72 \mathrm{~h}$, the $I R F-1$ silent group with different concentration all promoted proliferation and inhibited apoptosis $(\mathrm{P}<0.05)$ (Figure 2). These results suggested that the negative regulatory role of IFN- $\gamma$ in hematopoiesis may be completely reversed with the prolonged duration after silencing the expression of the $I R F-1$ gene. In this study, the proliferation ratio of the two cells at different time points was compared. The rate of proliferation of 32D cells of wild-type Akt was significantly higher $(\mathrm{P}<0.01)$ than that of inactive-type Akt. On the other hand, the apoptosis rate in wild-type was significantly less $(\mathrm{P}<0.01)$ than that of inactive Akt at all time points, especially at $48 \mathrm{~h}$ (Figure 3). This suggested that the expression of active Akt promotes the proliferation of cells and inhibition of apoptosis, and the Akt signal pathway might play a critical role in the IFN- $\gamma$-mediated reversal of promoting cell proliferation.

\section{Levels of p-Stat 3 and p-Stat 5 were investigated by Western blot}

The result suggested that Akt can promote the p-Stat3 level, which might be critical in the reversal of IFN- $\gamma$. Moreover, it was a key gene in the reversal of IFN- $\gamma$ effect after IRF-1 silencing. Compared to the control group, 32D cells with inactive-type Akt or wild-type Akt, the p-Stat5 level was not significantly different before the $I R F-1$ silencing in each group. The p-Stat5 level was significantly inhibited after $I R F-1$ silencing at the high concentration of IFN- $\gamma$. These observations revealed that IFN- $\gamma$ did not affect the p-Stat 5 level before IRF-1 silencing. Nevertheless, IFN- $\gamma$ significantly reduced the p-Stat 5 level at a specific concentration, thereby indicating that the reversal effect of high concentration of IFN- $\gamma$ might be achieved by reducing the p-Stat5 level after IRF-1 gene was silenced (Figure 4).

\section{Discussion}

\section{The role of IRF-1 gene in promoting apoptosis of IFN- $\gamma$}

Previous studies had shown that the excessive apoptosis of hematopoietic cells was caused by a high concentration of IFN- $\gamma$ in AA patients, the primary cause of AA incidence 
A

Merge
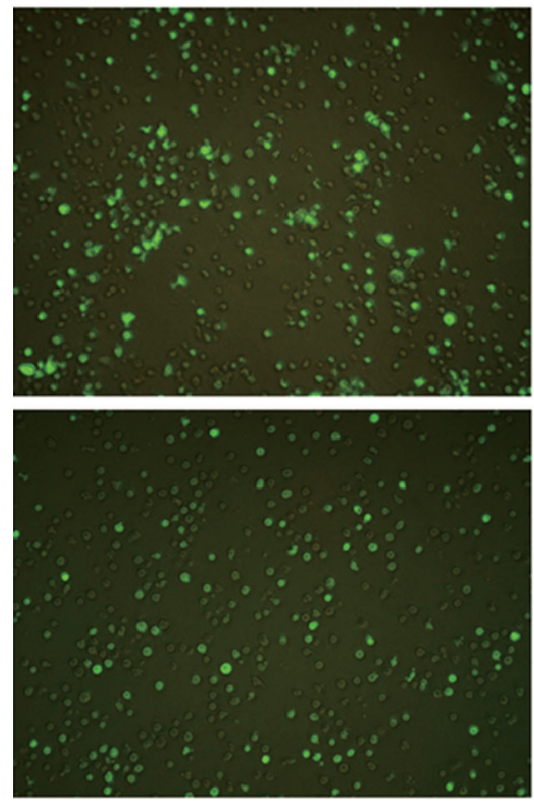

Bright field
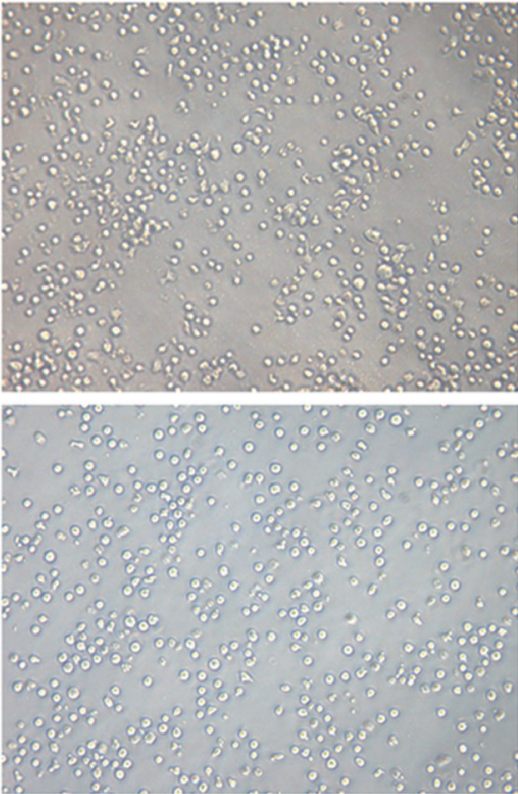

Fluorescent
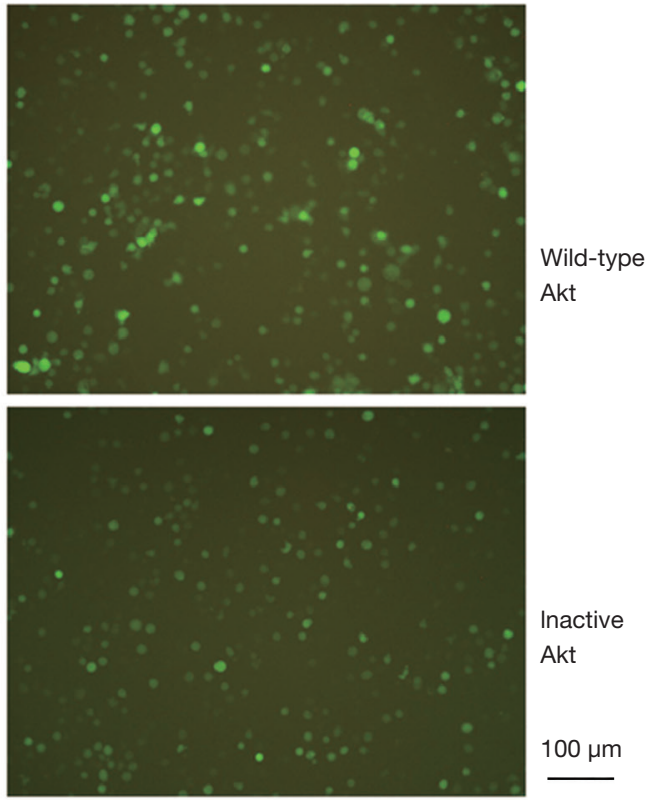

B

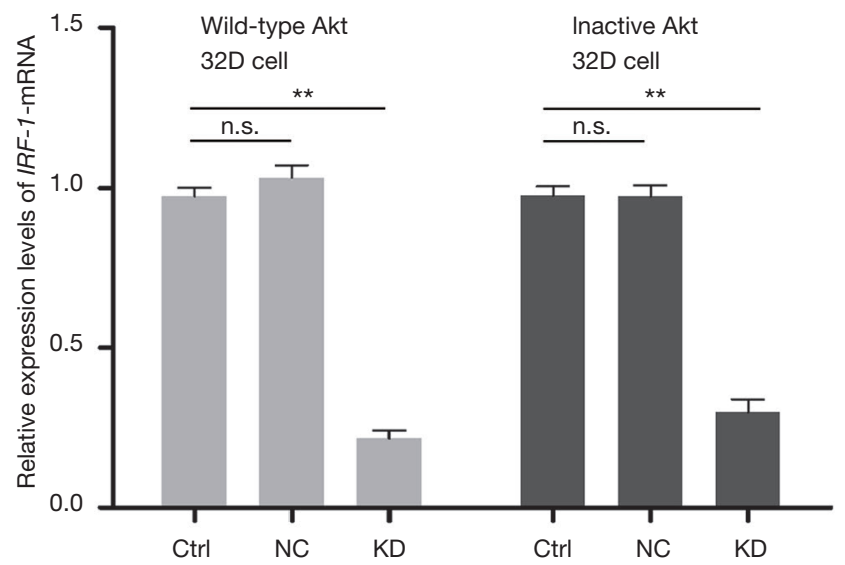

C

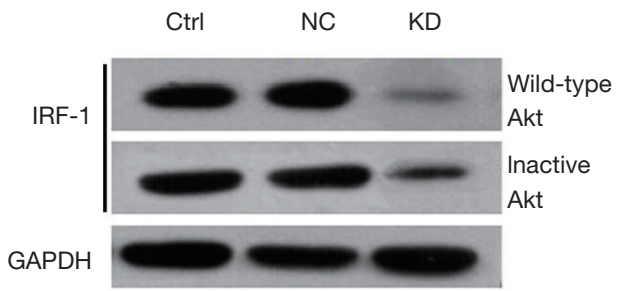

Figure 1 Transfection of IRF-1-siRNA plasmid and verification of IRF-1 gene silencing effect. (A) Modified-cell morphology observed with fluorescence microscope. 32D cells transfected with IRF-1-siRNA expressing wild-type Akt or inactive Akt. The transfection efficiency was $>80 \%$. (B) The expression levels of IRF-1 protein in 32D cells. The expression levels of IRF-1 protein in IRF-1-siRNA plasmid transfection group were significantly lower than that in the negative control and blank control groups in 32D cells expressing wild-type Akt (P<0.05). In the other $32 \mathrm{D}$ cells expressing the inactive-type Akt, the expression levels of IRF-1 protein in the IRF-1-siRNA plasmid transfection group were significantly lower than that in the negative control and blank control groups $(\mathrm{P}<0.05)$. (C) The relative expression levels of $I R F-1$-mRNA in the knockdown group were less than that in the empty vector negative control and blank control groups. The relative expression levels of $I R F-1$ mRNA in the knockdown group of 32D cells expressing wild-type Akt was $0.247 \pm 0.051$ that was significantly lower than the negative control and blank control groups $(\mathrm{P}<0.05)$. Moreover, in the case of 32D cells with inactive-type Akt, the $I R F-1 \mathrm{mRNA}$ expression levels in the knockdown group were $0.283 \pm 0.060$ that were markedly lower than the negative control and the blank control groups $(\mathrm{P}<0.05)$. ** $\mathrm{P}<0.01$; n.s., no significance; Ctrl, the blank control group; NC, the empty vector negative control; KD, the knockdown group transfected with IRF-1-siRNA plasmid; Akt, protein kinase B; IRF-1, interferon regulatory factor 1; mRNA, messenger RNA; GAPDH, glyceraldehyde-3-phosphate dehydrogenase. 
A

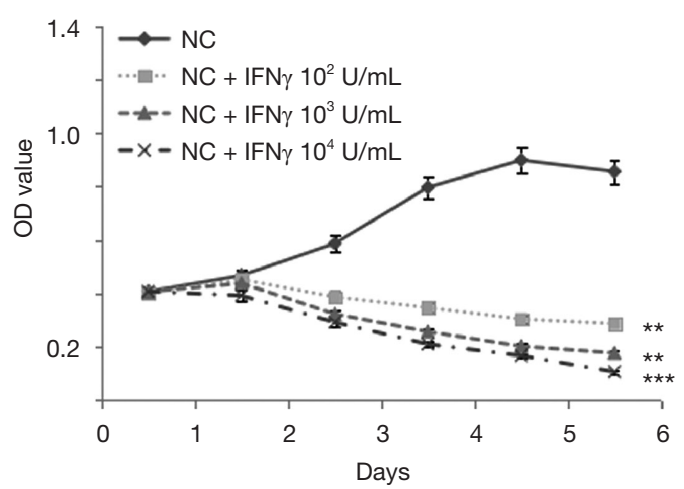

C

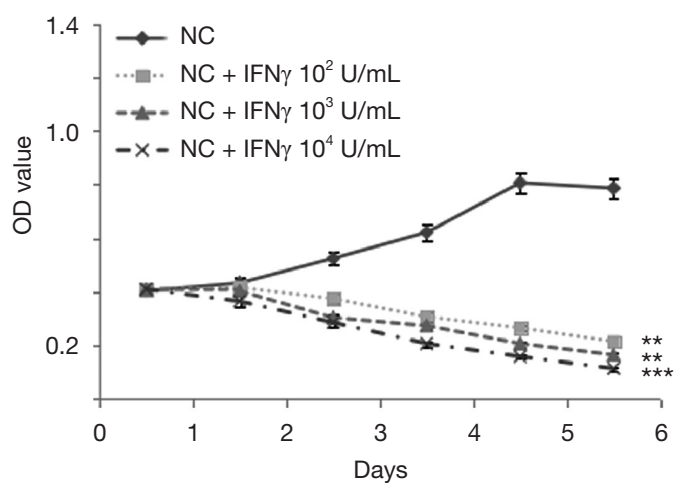

B

Wild-type Akt 32D cell

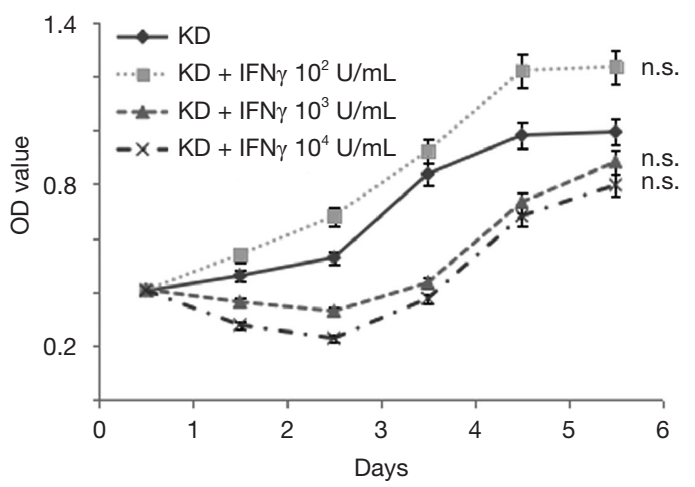

D Inactive Akt 32D cell

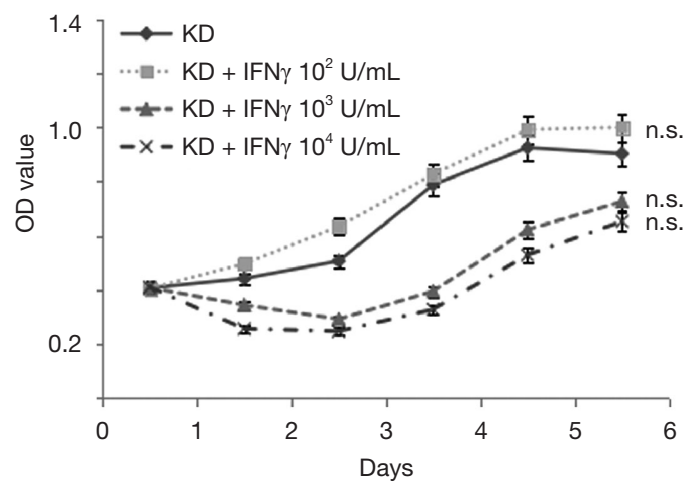

Figure 2 Effect of IFN- $\gamma$ on the proliferation and survival of 32D cells in the presence of various drug concentrations in transfection groups at different time points. The OD values of 32D cells of wild-type Akt (A,B) and inactive-type Akt $(\mathrm{C}, \mathrm{D})$ were measured and a linear correlation was identified between the OD value and the cell number. NC represents the empty vector negative control, and KD represents the knockdown group transfected with IRF-1-siRNA plasmid. The cell viability was tested at the indicated times using an MTS cell proliferation assay. The data represent the mean $\pm \mathrm{SD}$ of three independent experiments. ${ }^{* * *}, \mathrm{P}<0.001 ;{ }^{* *}, \mathrm{P}<0.01$; n.s., no significance; $\mathrm{NC}$, the empty vector negative control; KD, the knockdown group transfected with IRF-1-siRNA plasmid; OD, optical density; IFN- $\gamma$, interferon-gamma; Akt, protein kinase B.

$(1,2,10,11)$. The mechanism underlying IFN- $\gamma$-inhibited hematopoiesis was complex, involving several ways for the occurrence of AA. Several investigations indicated that $I R F-1$ gene could activate not only the expression of IFNs but also show the activity of tumor suppressor, which can inhibit cell proliferation as an anti-cancer effect (11-13); also, the micro-expression of IRF-1 inhibited the cell proliferation (3). These studies showed that $I R F-1$ gene plays a major role in the IFN- $\gamma$-inhibited cell proliferation. Sato et al. found that IFN- $\gamma$ promoted cell proliferation in leukemia when the expression of the IRF-1 protein was suppressed, and the proliferation efficiency was associated with the degree of $I R F-1$ gene inhibition (14-16). Herein, siRNA technology was employed to silence the IRF-1 gene, and the results suggested that the pro-apoptotic role of IFN- $\gamma$ reversed the cell proliferation and effectuated antiapoptosis after $I R F-1$ gene silencing. Thus, the critical role of $I R F-1$ gene in promoting apoptosis of IFN- $\gamma$ was evident.

\section{The role of Akt in promoting cell proliferation}

Akt is a downstream critical target protein of PI3K family. 


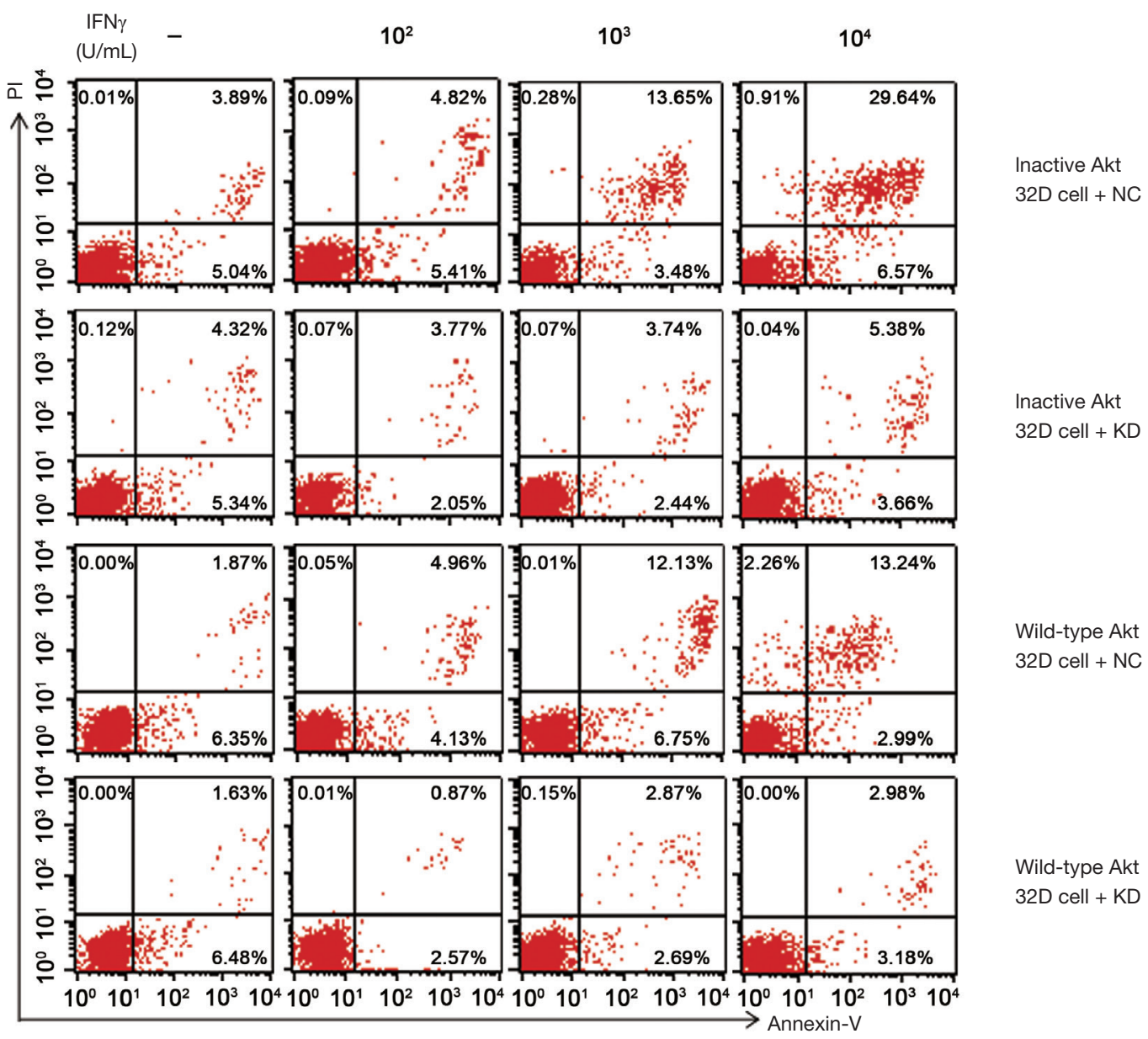

Figure 3 Flow cytometric analysis of apoptosis induced by IFN- $\gamma$ in 32D cells with wild-type Akt and inactive-type Akt. These cells were cultured in medium containing various concentrations of IFN- $\gamma$ for $48 \mathrm{~h}$, respectively, followed by analysis of apoptosis. The data shown are representative of three independent and statistically reproducible experiments. NC, the empty vector negative control; KD, the knockdown group transfected with IRF-1-siRNA plasmid; PI, propidium iodide; IFN- $\gamma$, interferon-gamma; Akt, protein kinase B.

It is commonly associated with in vivo signal transduction and is closely related to the cellular activity, metabolism regulation, and the inhibition of apoptosis (17-19). Our previous study suggested that Akt is involved in IFN- $\gamma$ mediated cell proliferation (20). To further confirm that the vital role of Akt in the effect of IFN- $\gamma$ before and after silencing the $I R F-1$ gene, the mechanism underlying IFN- $\gamma$ in the reversal of the effect was explored. 32D cells expressing wild-type of Akt as target cells and inactive Akt as control cells were used for comparing the diverse roles of IFN- $\gamma$. These results implied that the proliferation ratio of groups expressing wild-type Akt cells was greater than the corresponding groups expressing inactive the Akt cells at different time points and different concentrations of
IFN- $\gamma$. Consequently, the apoptosis rate was less than the corresponding cells, thereby indicating that the expression of active Akt promoted cell proliferation, and Akt signaling pathways played a key role in IFN- $\gamma$-promoted cell proliferation.

\section{The role of p-Stat 3 after IRF-1 gene silencing with and without IFN- $\gamma$}

IFN- $\gamma$ plays a role through multiple signal transduction pathways, and Jak-Stat is one of the classical signaling pathways (21-23). Signal transducers and activators of transcription (Stats) are transcription factors, composed of Stat1-4, Stat5a, Stat5b, and Stat6 in the cytoplasm and 


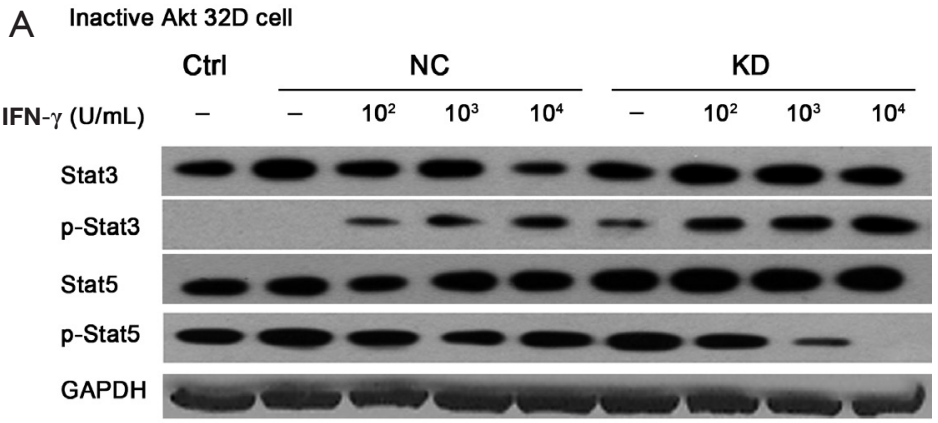

\section{B Wild-type Akt 32D cell}

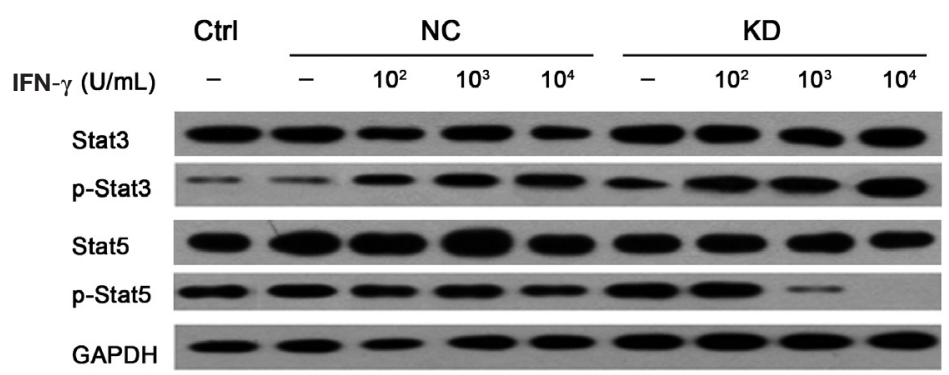

Figure 4 The expression of proteins from 32D cells in different plasmid-transfected groups and concentrations of IFN- $\gamma$. (A) The levels of Stat3, Stat5, p-Stat3 and p-Stat5 in 32D cells expressing inactive Akt. The phosphorylated Stat3 was up-regulated by IFN- $\gamma$ in a dosedependent manner. Blocking IRF-1 also stimulated the phosphorylated Stat3, and IFN- $\gamma$ further increased the p-Stat3 level. (B) The levels of Stat3, Stat5, p-Stat3 and p-Stat5 in 32D cells expressing wild-type Akt and the phosphorylated Stat5 in each group was similar before IRF-1 silencing. Only the high concentration of IFN- $\gamma$ significantly inhibited the phosphorylated Stat5 after IRF-1 silencing. Moreover, 32D cells with inactive Akt showed that the p-Stat3 level was less than that of the wild-type Akt. Ctrl, the blank control group; NC, the empty vector negative control; KD, the knockdown group transfected with IRF-1-siRNA plasmid; IFN- $\gamma$, interferon-gamma; Akt, protein kinase B; Stat3, the signal transducer and activator of transcription 3; Stat5, the signal transducer and activator of transcription 5; p-Stat3, phosphorylated Stat3; p-Stat5, phosphorylated Stat5; GAPDH, glyceraldehyde-3-phosphate dehydrogenase.

two with the signal pathway for tyrosine phosphorylation. Stat 3 is an essential member of the Stats family that is activated by phosphorylation. Also, it regulates the gene transcription when activated in the nucleus, followed by regulating cell proliferation, apoptosis, and angiogenesis (24-27). In this study, Western blotting was used to detect the p-Stat 3 level after $I R F-1$ gene silencing with and without IFN- $\gamma$. The results suggested that IFN- $\gamma$ promoted the phosphorylated Stat 3 , irrespective of $I R F-1$ silencing. In addition, the p-Stat 3 level was enhanced with an increase in the concentration of IFN- $\gamma$. The phosphorylated Stat 3 was raised in the reversal effect of IFN- $\gamma$, which played a critical role in reversing the effect of IFN- $\gamma$ after $I R F-1$ gene silencing.

\section{The level and role of p-Stat 5 after IRF-1 gene silencing}

Furthermore, some studies demonstrated that Stat5specific binding sequence existed in the promoter of $B c l-X L$, cyclin D1, $c-m y c$, and $I G F-I$ genes, and activated Stat 5 was imported to the nucleus, thereby inducing the expression of these genes to effectuate anti-apoptosis and promote cell proliferation (28-32). In addition, the study by Schepers et al. silenced Stat5 by RNAi and found that colony formation ability of hematopoietic stem and progenitor cells was significantly decreased (33). Interestingly, p-Stat5 exerted an anti-apoptosis role. To identify whether p-Stat 5 was related to the reversal role of IFN- $\gamma$ after $I R F-1$ silencing, we detected the expression of $\mathrm{p}$-Stat5 
by Western blotting pre- and post-silencing of IRF-1. These results suggested that the phosphorylated Stat5 was reduced at high concentrations of IFN- $\gamma$ when IRF-1 was silenced, while the experimental results of proliferation and apoptosis indicated that the high concentration of IFN- $\gamma$ promotes cell proliferation and inhibits apoptosis after IRF-1 silencing. Conversely, the decreased level of pStat5 inhibited apoptosis, which suggested its proapoptotic role, and the high concentration of IFN- $\gamma$ reversal effect might be achieved by downregulating the p-Stat 5 level. In addition, $I R F-1$ silencing might reverse some of the functions of IFN- $\gamma$, such that it would cause the other signaling pathways to decrease the p-Stat5 level, thereby promoting the cell growth. Strikingly, the groups of IRF- 1 RNAi at low concentration of IFN- $\gamma$ indicate the healthy state of cells with a low rate of apoptosis rate and no reduction in the p-Stat5 level. Thus, we speculated that the effect of proliferation in the presence of low concentration of IFN- $\gamma$ might not affect p-Stat5. Meanwhile, we found that the level of p-Stat 5 decreased, but Stat5 did not change, so IFN- $\gamma$ might induce a specific decrease in the process of Stat5 dephosphorylation. The previous experimental results and the growth-promoting effect of low concentrations of IFN- $\gamma$ together may be implemented through the Akt signaling pathway $(20,33)$. Furthermore, p-Stat 5 is speculated to exert a double modulatory role, and the effect of IFN- $\gamma$ is closely related to IRF-1 (34-37). Finally, the reversal mechanisms of the high and low concentrations of IFN- $\gamma$ were not found to be consistent, and $\mathrm{p}-\mathrm{Stat} 3$, Akt, and p-Stat 5 were involved in the reversal effect of IFN- $\gamma$. However, there are some limitations in our present study. We did not detect proteins related to Akt signaling pathway regulation, such as PRAS40, 4EBP and S6K. PRAS40 participates in regulating many signaling pathways such as mTORC1, Akt, NF-kB and RPL11 (38). Also, we didn't identify the isoform of the Akt involved in IFN- $\gamma$-mediated cell proliferation due to the limitations of the study, and we speculated that Akt 1 may be involved in IFN- $\gamma$-mediated cell proliferation based on literature reports (39). Owing to the complexity of the regulation of cytokine interactions and signal network, the mechanisms underlying the reversal of IFN- $\gamma$ necessitate further investigation, especially to identify whether the decrease in p-Stat5 is necessary for the function involved in the mechanism or it is independent.

\section{Acknowledgments}

We are grateful to Dr. F. Dong for providing the 32D cells expressing the wild-type and inactive Akt.

Funding: This study was funded by China Postdoctoral Science Foundation (Grant No. 2020M670038ZX) and Key Discipline of "Science \& Teaching Assisted Health Project" in Jiangsu province (Grant No. ZDXKB2016009).

\section{Footnote}

Reporting Checklist: The authors have completed the MDAR checklist. Available at http://dx.doi. org/10.21037/tcr-20-1866

Data Sharing Statement: Available at http://dx.doi. org/10.21037/tcr-20-1866

Conflicts of Interest: All authors have completed the ICMJE uniform disclosure form (available at http://dx.doi. org/10.21037/tcr-20-1866). The authors have no conflicts of interest to declare.

Ethical Statement: The authors are accountable for all aspects of the work in ensuring that questions related to the accuracy or integrity of any part of the work are appropriately investigated and resolved.

Open Access Statement: This is an Open Access article distributed in accordance with the Creative Commons Attribution-NonCommercial-NoDerivs 4.0 International License (CC BY-NC-ND 4.0), which permits the noncommercial replication and distribution of the article with the strict proviso that no changes or edits are made and the original work is properly cited (including links to both the formal publication through the relevant DOI and the license). See: https://creativecommons.org/licenses/by-nc-nd/4.0/.

\section{References}

1. Young NS, Calado RT, Scheinberg P. Current concepts in the pathophysiology and treatment of aplastic anemia. Blood 2006;108:2509-19.

2. Zeng W, Miyazato A, Chen G, et al. Interferon-gammainduced gene expression in CD34 cells: indentication of pathologic cytokine-specific signature profiles. Blood 2006;107:167-75.

3. Arockiaraj J, Sathyamoorthi A, Kumaresan V, et al. A murrel interferon regulatory factor-1: molecular characterization, gene expression and cell protection activity. Mol Biol Rep 2014;41:5299-309. 
4. Robertson SJ, Lubick KJ, Freedman BA, et al. Tickborne flaviviruses antagonize both IRF-1 and type I IFN signaling to inhibit dendritic cell function. J Immunol 2014;192:2744-55.

5. Lin YC, Lu MC, Lin C, et al. Activation of IFN- $\gamma / \mathrm{STAT} /$ IRF-1 in hepatic responses to Klebsiella pneumoniae infection. PLoS One 2013;8:e79961.

6. Sun $W, W u Z$, Lin Z, et al. Macrophage TNF- $\alpha$ licenses donor $\mathrm{T}$ cells in murine bone marrow failure and can be implicated in human aplastic anemia. Blood 2018;132:2730-43.

7. Tong LG, Liu H, Lin Z, et al. The Effects of IFN- $\gamma$ on AKT activated 32D Cells and its Mechanisms. Zhonghua Xue Ye Xue Za Zhi 2010;31:826-9.

8. Yu M, Li W, Luo S. Folic acid stimulation of neural stem cell proliferation is associated with altered methylation profile of PI3K/Akt/CREB. J Nutr Biochem 2014;25:496-502.

9. Li Z, Shen J, Wu WK, et al. Leptin induces cyclin D1 expression and proliferation of human nucleus pulposus cells via JAK/STAT, PI3K/Akt and MEK/ERK pathways. PLoS One 2012;7:e53176.

10. Wang $X$, Lin Z, Liu H, et al. The traditional Chinese medicine Lulongzaisheng decoction may affect the expression of Foxp3 by regulation of Akt and Stat3 phosphorylation in CD4+CD25+ regulatory T cells from severe aplastic anemia mouse models. Zhonghua Xue Ye Xue Za Zhi 2015;36:689-91.

11. Miller CH, Maher SG, Young HA. Clinical Use of Interferon-gamma. Ann N Y Acad Sci 2009;1182:69-79.

12. Stang MT, Armstrong MJ, Watson GA, et al. Interferon regulatory factor-1-induced apoptosis mediated by a ligand-independent fas-associated death domain pathway in breast cancer cells. Oncogene 2007;26:6420-30.

13. Luo S, Chen Y, Zhao L, et al. Effect of nutritional supplement on bone marrow-derived mesenchymal stem cells from aplastic anaemia. Br J Nutr 2018;119:748-58.

14. Murtas D, Maric D, De Giorgi V, et al. IRF-1 responsiveness to IFN- $\gamma$ predicts different cancer immune phenotypes. Br J Cancer 2013;109:76-82.

15. Li P, Du Q, Cao Z, et al. Interferon-gamma induces autophagy with growth inhibition and cell death in human hepatocellular carcinoma (HCC) cells through interferonregulatory factor-1 (IRF-1). Cancer Lett 2012;314:213-22.

16. Sato T, Selleri C, Young NS, et al. Inhibition of Interferon regulatory factor-1 expression results in predominance of cell growth stimulatory effects of Interferon- $\gamma$ due to phosphorylation of stat1 and stat3. Blood 1997;90:4749-58.
17. Lee DS, Ko W, Kim DC, et al. Cudarflavone B Provides Neuroprotection against Glutamate-Induced Mouse Hippocampal HT22 Cell Damage through the Nrf2 and PI3K/Akt Signaling Pathways. Molecules 2014;19:10818-31.

18. Watanabe T, Nakamura S, Ono T, et al. Pyrrolidinium fullerene induces apoptosis by activation of procaspase- 9 via suppression of Akt in primary effusion lymphoma. Biochem Biophys Res Commun 2014;451:93-100.

19. Di Buduo CA, Currao M, Pecci A, et al. Revealing eltrombopag's promotion of human megakaryopoiesis through AKT/ERK-dependent pathway activation. Haematologica 2016;101:1479-88.

20. Liu H, Mihara K, Tanaka $H$, et al. Interferon- $\gamma$ attenuates the survival activity of G-CSF through PI3K/Akt signaling pathway in mouse multipotent progenitor cells. Ann Hematol 2007;86:547-55.

21. Kim J, Yoon Y, Jeoung D, et al. Interferon- $\gamma$ stimulates human follicular dendritic cell-like cells to produce prostaglandins via the JAK-STAT pathway. Mol Immunol 2015;66:189-96.

22. Park MS, Kim BR, Kang S, et al. The antihypertension drug doxazosin suppresses JAK/STATs phosphorylation and enhances the effects of IFN- $\alpha / \gamma$-induced apoptosis. Genes Cancer 2014;5:470-9.

23. Dwane L, Gallagher WM, Ní Chonghaile T, et al. The Emerging Role of Non-traditional Ubiquitination in Oncogenic Pathways. J Biol Chem 2017;292:3543-51.

24. Stark GR, Darnell JE Jr. The JAK-STAT pathway at twenty. Immunity 2012;36:503-14.

25. Pu X, Guo QX, Long HA, et al. Effects of mTOR-STAT3 on the migration and invasion abilities of hepatoma cell and mTOR-STAT3 expression in liver cancer. Asian Pac J Trop Med 2014;7:368-72.

26. Zhang P, Li H, Yang B, et al. Biological significance and therapeutic implication of resveratrol-inhibited Wnt, Notch and STAT3 signaling in cervical cancer cells. Genes Cancer 2014;5:154-64.

27. McLemore ML, Grewal S, Liu F, et al. STAT-3 activation is required for normal G-CSF-dependent proliferation and granulocytic differentiation. Immunity 2001;14:193-204.

28. Schuringa JJ, Wu K, Morrone G, et al. Enforced activation of STAT5A facilitates the generation of embryonic stemderived hematopoietic stem cells that contribute to hematopoiesis in vivo. Stem Cells 2004;22:1191-204.

29. Chia DJ, Ono M, Woeme J, et al. Characterization of distinct Stat $5 \mathrm{~b}$ binding sites that mediate growth hormone-stimulated IGF-I gene transcription. J Biol 
Chem 2006;281:3190-97.

30. Sehgal PB. Non-genomic STAT5-dependent effects at the endoplasmic reticulum and Golgi apparatus and STAT6GFP in mitochondria. Jakstat 2013;2:e24860.

31. Wang X, Zeng J, Shi M, et al. Targeted blockage of signal transducer and activator of transcription 5 signaling pathway with decoy oligodeoxynucleotides suppresses leukemic K562 cell growth. DNA Cell Biol 2011;30:71-8.

32. Turkson J. STAT proteins as novel targets for cancer drug discovery. Expert Opin Ther Targets 2004;8:409-22.

33. Schepers H, Gosliga D, Wierenga AT, et al. STAT5 is required for long-term maintenance of normal and leukemic human stem/progenitor cells. Blood 2007,110:2880-8.

34. Aronis KN, Chamberland JP, Mantzoros CS. GLP-1 promotes angiogenesis in human endothelial cells in a dose-dependent manner, through the Akt, Src and PKC pathways. Metabolism 2013;62:1279-86.

Cite this article as: Lin Y, Zhang R, Jiang S, Lu W, Lin Z, Liu H. Effects of IFN- $\gamma$ on the proliferation of 32D cells expressing Akt after IRF-1 gene silencing. Transl Cancer Res 2021;10(1):327336. doi: $10.21037 /$ tcr-20-1866
35. Asao H, Fu XY. Interferon-gamma has dual potentials in inhibiting or promoting cell proliferation. J Biol Chem 2000;275:867-74.

36. Sheng $W$, Yang F, Zhou Y, et al. STAT5 programs a distinct subset of GM-CSF-producing T helper cells that is essential for autoimmune neuroinflammation. Cell Res 2014;24:1387-402.

37. Akada H, Akada S, Hutchison RE, et al. Critical role of Jak2 in the maintenance and function of adult hematopoietic stem cells. Stem Cells 2014;32:1878-89.

38. Lv D, Liu J, Guo L, et al. PRAS40 deregulates apoptosis in Ewing sarcoma family tumors by enhancing the insulin receptor/Akt and mTOR signaling pathways. Am J Cancer Res 2016;6:486-97.

39. Chen WS, Xu PZ, Gottlob K, et al. Growth retardation and increased apoptosis in mice with homozygous disruption of the Akt1 gene. Genes Dev 2001;15:2203-8. 